\title{
A broad-range survey of ticks from livestock in Northern Xinjiang: changes in tick distribution and the isolation of Borrelia burgdorferi sensu stricto
}

Yuan-Zhi Wang ${ }^{1+}$, Lu-Meng Mu ${ }^{1+}$, Ke Zhang ${ }^{2+}$, Mei-Hua Yang ${ }^{3+}$, Lin Zhang ${ }^{4+}$, Jing-Yun Du ${ }^{1+}$, Zhi-Qiang Liu, ${ }^{5,6 \dagger}$, Yong-Xiang Li ${ }^{1+}$, Wei-Hua Lu ${ }^{5}$, Chuang-Fu Chen ${ }^{1,2^{*}}$, Yan Wang ${ }^{1}$, Rong-Gui Chen ${ }^{7}$, Jun Xu ${ }^{8}$, Li Yuan ${ }^{1}$, Wan-Jiang Zhang ${ }^{1}$, Wei-Ze Zuo ${ }^{9}$ and Ren-Fu Shao ${ }^{10}$

\begin{abstract}
Background: Borreliosis is highly prevalent in Xinjiang Uygur Autonomous Region, China. However, little is known about the presence of Borrelia pathogens in tick species in this region, in addition Borrelia pathogens have not been isolated from domestic animals.

Methods: We collected adult ticks from domestic animals at 19 sampling sites in 14 counties in northern Xinjiang from 2012 to 2014. Ticks were identified to species by morphology and were molecularly analysed by sequences of mitochondrial 165 rDNA gene; 4-8 ticks of each species at every sampling site were sequenced. 112 live adult ticks were selected for each species in every county, and were used to culture Borrelia pathogens; the genotypes were then determined by sequences of the 5S-23S rRNA intergenic spacer and the outer surface protein A (ospA) gene.
\end{abstract}

Results: A total of 5257 adult ticks, belonging to four genera and seven species, were collected. Compared with three decades ago, the abundance of the five common tick species during the peak ixodid tick season has changed. Certain tick species, such as Rhipicephalus turanicus (Rh. turanicus), was found at Jimusaer, Yining, Fukang, and Chabuchaer Counties for the first time. Additionally, the sequence analyses showed that the Hyalomma asiaticum (Hy. asiaticum), Haemaphysalis punctata (Ha. punctata), and Dermacentor marginatus (D. marginatus) that were collected from different sampling sites ( $\geq 3$ sites) shared identical $16 \mathrm{~S} \mathrm{rDNA}$ sequences respectively. For the tick species that were collected from the same county, such as Hy. asiaticum from Shihezi County and Rh. turanicus from Yining County, their $16 \mathrm{~S}$ rDNA sequences showed genetic diversity. In addition, sixteen Borrelia isolates were found in Hy. asiaticum, Ha. punctata, D. marginatus and Rh. turanicus, which infested cattle, sheep, horse and camel in Yining, Chabuchaer, Shihezi and Shawan Counties. All of the isolates were genetically identified as B. Burgdorferi sensu stricto.

(Continued on next page)

\footnotetext{
* Correspondence: chuangfuchen666@126.com

${ }^{\dagger}$ Equal contributors

'School of Medicine, Shihezi University, 832003 Shihezi, Xinjiang, Peoples

Republic of China

${ }^{2}$ Institute of Agricultural and Rural Development, Pingdingshan University,

467000 Pingdingshan, Henan, Peoples Republic of China

Full list of author information is available at the end of the article
}

\section{Ciomed Central}

(C) 2015 Wang et al. Open Access This article is distributed under the terms of the Creative Commons Attribution 4.0 International License (http://creativecommons.org/licenses/by/4.0/), which permits unrestricted use, distribution, and reproduction in any medium, provided you give appropriate credit to the original author(s) and the source, provide a link to the Creative Commons license, and indicate if changes were made. The Creative Commons Public Domain Dedication waiver (http://creativecommons.org/publicdomain/zero/1.0/) applies to the data made available in this article, unless otherwise stated. 
(Continued from previous page)

Conclusions: Warmer and wetter climate may have contributed to the altered distribution and abundance of the five most common ticks in northern Xinjiang. The genetic analyses showed that certain tick species, such as Hy. asiaticum or Rh. turanicus, exhibit genetic commonness or diversity. Additionally, this study is the first to isolate $B$. burgdorferi sensu stricto in Hy. asiaticum asiaticum, H. punctata, D. nuttalli and D. marginatus ticks from domestic animals. These ticks may transmit borreliosis among livestock.

Keywords: Tick species, Borrelia burgdorferi, Northern Xinjiang

\section{Background}

Lyme disease (borreliosis) is one of the most prevalent tick-borne zoonoses in eastern Asia [1]. One of its aetiologic agents, Borrelia burgdorferi sensu lato, is primarily distributed in northern China, whereas $B$. garinii and $B$. afzelii are distributed in northeastern and northwestern China [2]. In 2001, B. garinii and B. afzelii were firstly isolated from free-living Ixodes persulcatus in Xinjiang, China (XARC) [3]. Liu et al. reported that the blood samples of domestic animals in Xinjiang were highly positive (14\%) to Borrelia burgdorferi-like bacteria, as determined by polymerase chain reaction (PCR) [4], but the pathogens have not been isolated from ticks infecting livestock.

Xinjiang occupies one-sixth of China and borders eight countries with a 5,600-km long borderline. There are three major mountains in Xinjiang: Altay Mountain in the north, Tianshan Mountain in the middle, and Kunlun Mountain in the south. Between the mountains are two large basins, Junggar Basin in the north and Tarim Basin in the south [5]. High mountains, valleys, plains, Gobi desert, and other additional characteristics constitute the various landscapes of northern Xinjiang [6]. Additionally, this region is halfway along the old Silk Road between eastern Asia and Europe, and international livestock trade is frequent [3].

Forty-two species of ticks in nine genera have been identified in Xinjiang, which represent more than $1 / 3$ of the total tick species found in China [7]. According to Kong et al., Ixodes persulcatus (I. persulcatus), Dermacentor nuttalli (D. nuttalli), Hy. asiaticum asiaticum, D. marginatus, and Dermacentor niveus (D. niveus) were the five dominant tick species during the peak ixodid tick season 30 years ago [8]. Since then, there have been few comprehensive reports regarding the distribution and abundance of ticks in northern Xinjiang.

In the present study, we investigated the distribution and abundance of ticks from livestock in northern Xinjiang. We also isolated Borrelia pathogens from ticks for the first time in this region.

\section{Methods}

\section{Sampling area}

We established 19 sampling sites in 14 counties in northern Xinjiang-Jimusaer, Qitai, Mulei, Fukang, Miquan,
Chanagji, Shihezi, Shawan, Karamay, Fuhai, Qinghe, Tacheng, Yining and Chabuchaer, from 2012 to 2014, in May when the peak activities of adult ticks occur in northern Xinjiang. The first 10 counties are primarily livestock husbandry regions, and the 4 remaining counties include 4 trade ports (Takesh Ken, Baketu, Yining, and Dulata) that are adjacent to Mongolia and Kazakhstan and are important trade zones for livestock and livestock products (Fig. 1). In accordance with the different types of landscapes, 1-4 sampling sites were selected in each county. Geographic information regarding the tick habitats and the geographic coordinates, altitude, and plant distributions of all 33 sampling sites were shown in Additional file 1: Table S1. The annual precipitation and average annual temperatures from 1962 to 2012 were shown in Fig. 2.

The livestock reared in these regions are mainly autochthonous. The vast majorities (approximately $95 \%$ ) of the sheep are Kazakh, Chinese Merino, Hu, and Altay breeds; the majority of the cattle are Xinjiang Brown, Holstein and Kazakh breeds. The horses are the Kazakh breed and the camels are the Bactrian breed. All of the livestock we checked for ticks were grazed in the traditional manner; no chemical acaricides were used prior to the tick sampling.

\section{Tick sampling and identification}

Adult ticks were collected over three consecutive years. In 2012, the ticks were collected from 22 livestock flocks at 12 sampling sites in 10 counties. In 2013, seven livestock flocks at seven sampling sites in 5 counties were surveyed; these sampling sites are in various landscapes. In 2014, four livestock flocks in four counties were surveyed. The information regarding all of the collected specimens, including their location, host, number of ticks collected from the body of each animal and the date of collection, were recorded. The tick sampling was performed over the entire body of each animal, including sheep, cattle, horses, and camels, at different intervals, and the collected ticks were kept alive until they were transferred to the laboratory. The ticks collected were counted, and identified to species by morphology according to Walker et al. [9] and Estrada-Peňa et al. [10]. 


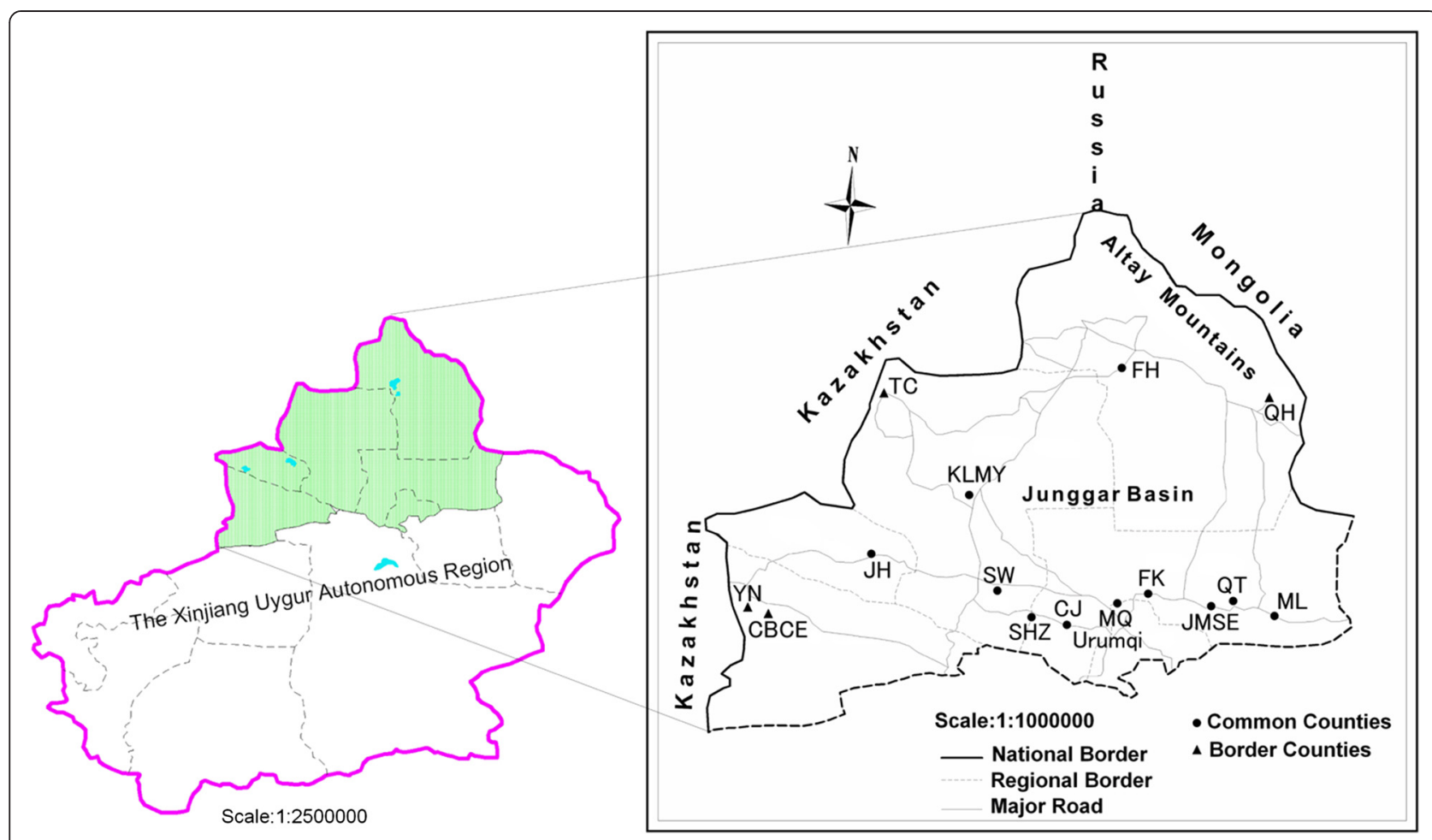

Fig. 1 A map of the study area. Left: the Xinjiang Uygur Autonomous Region. Right: the 14 surveyed counties in northern Xinjiang

\section{Genetic analysis}

After detailed morphological investigation, 110 representative tick specimens, with 1-4 ticks for each tick species at every sampling site, were used to analyse the genetic diversity. The genomic DNA extraction was performed using a commercial kit (DNeasy Blood \& Tissue
Kit, Qiagen GmbH, Hilden, Germany) according to the manufacturer's instructions. The $16 S$ rDNA mitochondrial gene sequences were then amplified according to the protocol of Black et al. [11]. The PCR products were purified using the TIANgel Midi Purification Kit (TIANGEN, Beijing, China) and then sequenced.
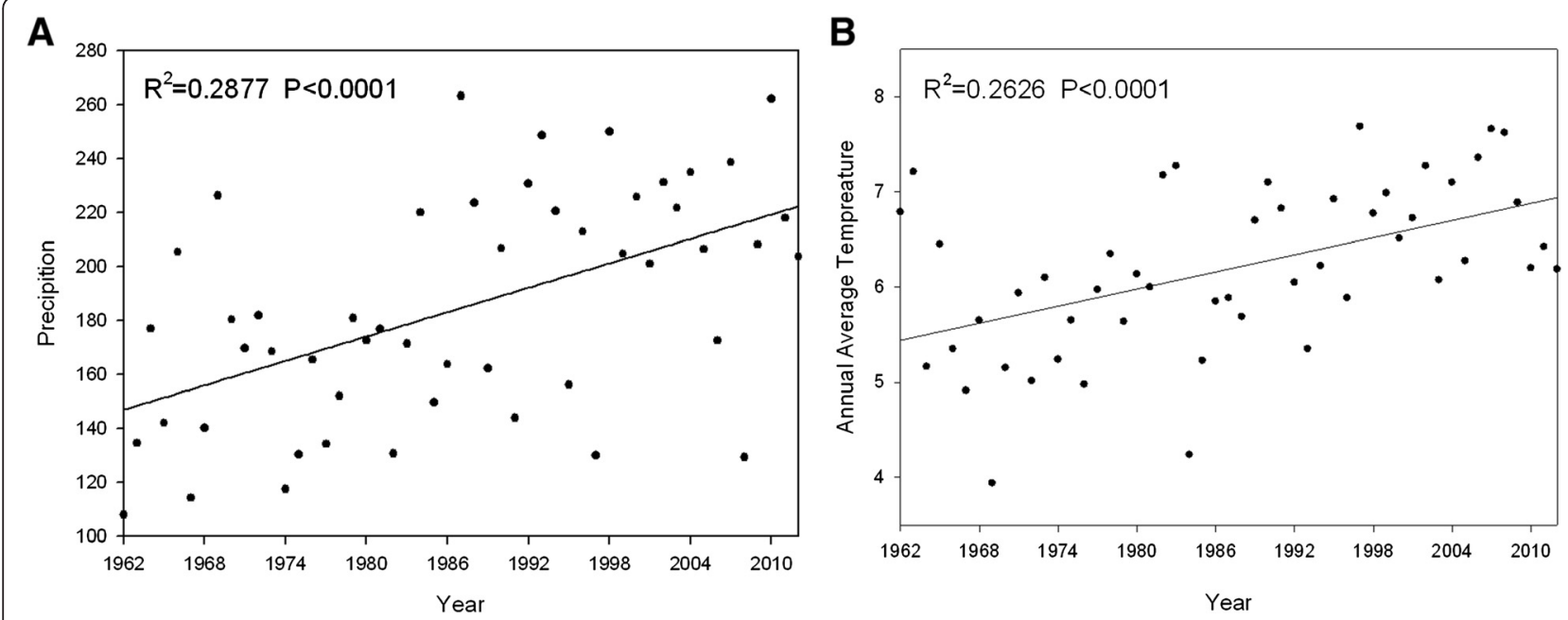

Fig. 2 The annual precipitation and average annual temperatures in northern Xinjiang from 1962-2012. The annual precipitation and average annual temperature are shown in (a) and (b), respectively. All the data came from eight international surveillance sites (Jinghe, Qitai, Altay, Fuyun, Hefeng, Karamay, Yining, and Urumqi) in northern Xinjiang and were collected from 1962-2012. The raw data are from the China Meteorological Data Sharing Service System (http://cdc.cma.gov.cn/) and were analyzed using Sigma Plot software 


\section{Isolation and identification of Borrelia pathogens}

A total of 112 live adult ticks, including 34 Hy. Asiaticum asiaticum, $39 \mathrm{H}$. punctata, $10 \mathrm{D}$. nuttalli, $8 \mathrm{D}$. marginatus, and 21 Rh. turanicus specimens, were collected from each county and were selected for the isolation and identification of Borrelia. The ticks were first dipped in $70 \%$ alcohol for approximately $1 \mathrm{~h}$ and dissected on sterile plates according to a method described by Burgdorfer et al. [12]. The midguts of each tick species from a county were then transferred to a tube containing $6 \mathrm{~mL}$ of BSK-H medium (Nunc, Roskilde, Denmark). All of the cultures were then incubated at $33{ }^{\circ} \mathrm{C}$ for up to 6 weeks and examined periodically using silver staining. Twenty-four samples were also photographed using a microscope (Model: BX60, OLYMPUS, Japan) equipped with a digital camera (Model: DP70, OLYMPUS, Japan) linked to a computer. Additionally, to determine the Borrelia genotype, the sequences of $5 S-23 S$ rRNA intergenic spacers and the outer surface protein A (OspA) gene were amplified and sequenced from positive cultures.

\section{Cycling conditions for the genetic analyses of the 110} representative tick specimens and the Borrelia isolates The detailed cycling conditions for $16 \mathrm{~S} r D N A$, the 5S-23S rRNA intergenic spacer, and OspA are described in the Additional file 2.

\section{Phylogenic analysis}

Data comparisons were performed using those tick species that had more than 10 available specimens. Sequence alignments to determine the nucleotide percentage from each species were performed using Clustal X 2.0 [13], and the phylogenic relationships among the representative tick specimens were inferred using MEGA5 [14]. A phylogenic tree was then constructed using the neighbor-joining method [15].

A total of 67 reference sequences, including sequences from the Dermanyssus gallinae (L34326.1) and Spinturnix myoti (FJ225960) outgroups, were used for the phylogenetic analysis, and 23 nucleotide sequences from our study have been deposited in the GenBank database (16S rDNA: KF547980, KF547981, KF547982, KF547983, KF547984, KF547985, KF547986, KF547987, KF547988, KF547989, KF547990, KF547991, KF547992, KF547993, KF547994, and KF547995; 5S-23S rDNA intergenic spacer of Borrelia: KF547996, KF547997, KF547998, KJ459337, KJ459338, KJ459339 and KJ459340).

\section{Results}

\section{Tick collection and morphological identification}

A total of 5257 adult ticks, from seven species and four genera, were collected from 19 sampling sites in 14 counties in northern Xinjiang (Table 1). Hy. asiaticum asiaticum (32.70 \%) was the most frequently collected species, followed by Ha. punctata (31.17 \%), D. nuttalli (13.47 \%), D. marginatus (12.10 \%), Rh. turanicus (9.53 \%), Hyalomma detritum (0.02\%), and Haemaphysalis concinna $(0.02 \%)$ (Table 1$)$.

\section{Molecular identification and analysis}

A phylogenetic tree based on $16 \mathrm{~S} r D N A$ sequences of the representative tick specimens is shown in Fig. 3. The results showed that Hy. asiaticum, Ha. punctata, and $D$. marginatus samples that were obtained from different sampling sites ( $\geq 3$ sites) shared common $16 \mathrm{~S}$ rDNA sequences. Their accession numbers are KF547992, KF547980, and KF547986, respectively. Additionally, the $16 \mathrm{~S} r D N A$ sequences of certain tick species from the same sampling site showed diversity. For example, 3 different sequences were obtained from $R h$. turanicus from Yining County, with the accession numbers KF547984, KF547987, and KF547989.

\section{Isolation and identification of Borrelia from ticks}

Sixteen positive cultures of Borrelia from Hy. Asiaticum asiaticum, Ha. punctata, D. marginatus, and Rh. turanicus collected in Yining, Chabuchaer, Shihezi and Shawan Counties were observed. Their OspA sequences showed $100 \%$ identity to the B. burgdorferi reference B31 strain (Accession NO: X63412). Their 5S-23S rRNA sequences showed that all of the positive culture extracts are $B$. Burgdorferi sensu stricto (Fig. 4).

\section{Discussion}

The distribution of ticks within a specific habitat depends on several environmental and climatic factors, such as annual rainfall, atmospheric temperature and relative humidity $(\mathrm{RH})$, vegetation cover, altitude and host availability. This study was conducted in May, from 2012 to 2014, when the $\mathrm{RH}$ and the atmospheric temperature provide an ideal environment for most tick species. Therefore, the abundance of adult ticks on livestock is expected to be at its peak. In the present study, the spatial distribution and abundance of ticks infesting livestock was assessed. The following phenomena were observed and compared with previous findings, especially with those from the investigation conducted by Kong et al. [8].

First, the abundance of I. persulcatus was reduced. In previous studies, I. persulcatus was found to be the dominant tick species in northern China, including Xinjiang, and the species was found distributed at altitudes of $1300-2120 \mathrm{~m}$ above sea level (m.a.s.l). In this study, I. persulcatus was not identified in the various analysed landscapes over a wide range of altitudes. When comparing the average monthly precipitation and temperatures from 1973 to 2012, we found that lower 
Table 1 The counts of each tick species from the 19 sampling sites between 2012 and 2014

\begin{tabular}{|c|c|c|c|c|c|c|c|c|c|}
\hline \multirow[t]{2}{*}{ Counties } & \multirow{2}{*}{$\begin{array}{l}\text { Flock } \\
\text { NO. } \\
\text { (year) }\end{array}$} & \multirow[t]{2}{*}{ Hosts } & \multicolumn{2}{|l|}{ Haemaphysalis } & \multicolumn{2}{|l|}{ Dermacentor } & \multicolumn{2}{|l|}{ Hyalomma } & \multirow{2}{*}{$\begin{array}{l}\text { Rhipicephalus } \\
\text { R. turanicus } \\
(\hat{\delta} / q)\end{array}$} \\
\hline & & & $\begin{array}{l}\text { H. punctata } \\
\left(\sigma^{2} / q\right)\end{array}$ & $\begin{array}{l}\text { H. conicinna } \\
(\hat{\delta} / P)\end{array}$ & $\begin{array}{l}\text { D. marginatus } \\
(\hat{\partial} / q)\end{array}$ & $\begin{array}{l}\text { D. nuttalli } \\
(\hat{\delta} / q)\end{array}$ & $\begin{array}{l}\text { H. asiaticum } \\
(\hat{\partial} / q)\end{array}$ & $\begin{array}{l}\text { H. detritum } \\
(\hat{\alpha} / \text { / })\end{array}$ & \\
\hline \multirow[t]{3}{*}{ Chabuchaer (CBCE) } & $1 \#(2013)$ & Sheep & & & & & & & $151(77 / 74)$ \\
\hline & 2\#(2013) & Sheep & & & & & & & $191(97 / 94)$ \\
\hline & $3 \#(2014)$ & Sheep & & & & & & & $69(32 / 37)$ \\
\hline Changji (CJ) & $1 \#(2012)$ & Cattle & & & & & $180(76 / 104)$ & & \\
\hline \multirow[t]{2}{*}{ Fuhai (FH) } & $1 \#(2012)$ & Sheep & $11(5 / 6)$ & & & $15(6 / 9)$ & $148(95 / 53)$ & & \\
\hline & 2\#(2012) & Sheep & $9(4 / 5)$ & & & $8(3 / 5)$ & $150(90 / 60)$ & & \\
\hline \multirow[t]{3}{*}{ Fukang (FK) } & $1 \#(2012)$ & Cattle, sheep & & & $9(6 / 3)$ & & $60(43 / 27)$ & & $16(12 / 4)$ \\
\hline & $2 \#(2012)$ & Cattle, sheep & $152(87 / 65)$ & & $161(80 / 81)$ & & & & \\
\hline & $3 \#(2012)$ & Cattle, sheep & & & & & $139(86 / 53)$ & & \\
\hline \multirow[t]{3}{*}{ Jimusaer (JMSE) } & $1 \#(2012)$ & Sheep & $76(54 / 22)$ & & & & & & \\
\hline & 2\#(2012) & Cattle, sheep & & & & $195(104 / 91)$ & $22(8 / 14)$ & & \\
\hline & 3\#(2012) & Camel & & & & & & & $74(11 / 63)$ \\
\hline \multirow[t]{2}{*}{ Karamay (KLMY) } & $1 \#(2012)$ & Cattle & & & & & $80(40 / 40)$ & & \\
\hline & 2\#(2012) & Cattle & $89(22 / 67)$ & & & & & & \\
\hline \multirow[t]{2}{*}{ Miquan (MQ) } & $1 \#(2012)$ & Cattle, sheep & & & & & $93(36 / 57)$ & & \\
\hline & 2\#(2012) & Cattle, sheep & & & $90(70 / 20)$ & & $112(48 / 64)$ & & \\
\hline \multirow[t]{3}{*}{ Mulei (ML) } & $1 \#(2012)$ & Cattle, sheep & $38(32 / 6)$ & & & $47(44 / 3)$ & & $1(0 / 1)$ & \\
\hline & 2\#(2012) & Cattle, sheep & & & & $97(65 / 32)$ & & & \\
\hline & 3\#(2012) & Cattle, sheep & & & & $196(112 / 84)$ & & & \\
\hline \multirow[t]{2}{*}{ Qinghe $(\mathrm{QH})$} & $1 \#(2012)$ & Sheep & $62(41 / 21)$ & & & & & & \\
\hline & 2\#(2014) & Sheep & $41(18 / 23)$ & & & & $136(66 / 70)$ & & \\
\hline \multirow[t]{4}{*}{ Qitai (QT) } & $1 \#(2012)$ & Cattle, sheep & $187(121 / 66)$ & & & & $219(160 / 59)$ & & \\
\hline & 2\#(2012) & Sheep & & & & $22(5 / 17)$ & & & \\
\hline & 3\#(2012) & Cattle, sheep & & & & & $48(12 / 36)$ & & \\
\hline & $4 \#(2012)$ & Sheep & & & & $65(41 / 24)$ & $29(23 / 6)$ & & \\
\hline \multirow[t]{3}{*}{ Shawan (SW) } & $1 \#(2012)$ & Sheep, horse & $100 / 76$ & & $30 / 19$ & & $47 / 29$ & & \\
\hline & 2\#(2013) & Sheep & $112(63 / 49)$ & & $87(57 / 30)$ & & $44(25 / 19)$ & & \\
\hline & 3\#(2013) & Sheep & $101(62 / 39)$ & & $83(55 / 28)$ & & $42(25 / 17)$ & & \\
\hline \multirow[t]{2}{*}{ Shihezi (SHZ) } & $1 \#(2013)$ & Cattle, sheep & $187 / 150$ & & $40 / 27$ & $21 / 14$ & $57 / 35$ & & \\
\hline & 2\#(2014) & Camel, sheep & $121(72 / 49)$ & & $54(31 / 23)$ & & $83(49 / 34)$ & & \\
\hline Tacheng (TC) & 1\#(2013) & Sheep & & $0 / 1$ & $23 / 13$ & & & & \\
\hline \multirow[t]{2}{*}{ Yining (YN) } & $1 \#(2013)$ & Cattle, Sheep & $48 / 58$ & & & & & & \\
\hline & 2\#(2014) & Cattle, Sheep & $73(32 / 41)$ & & & & & & \\
\hline Total number & & 5257 & $1691(948 / 743)$ & $1(0 / 1)$ & $636(392 / 244)$ & $708(418 / 244)$ & $1719(961 / 758)$ & $1(0 / 1)$ & $501(229 / 272)$ \\
\hline Percentage (\%) & & & $32.17 \%$ & $0.02 \%$ & $12.10 \%$ & $13.47 \%$ & $32.70 \%$ & $0.02 \%$ & $9.53 \%$ \\
\hline
\end{tabular}

summer precipitation levels coinciding with raised summer temperatures may inhibit the survival and development of I. persulcatus.

Second, Rh. turanicus has a tendency to shift northwards. It is primarily distributed in the plains and rural areas of the Shanxi, Guangxi, Hainan and Xinjiang Provinces in China and is the second most abundant tick species in the southern region of Xinjiang [16].
However, Rh. turanicus was first collected in the plains of Jimusaer, Yining, Fukang, and Chabuchaer Counties, in northern Xinjiang. By tracing the movement routes of $R h$. turanicus, we found that from 1973 to1985, the species was distributed below $25^{\circ} \mathrm{N}$, which then shifted northwards to the hilly terrain of Huocheng County $\left(44^{\circ} \mathrm{N}\right)$ in 1993 [17], and it is currently found in the semi-desert grasslands of Fukang County $\left(48^{\circ} \mathrm{N}\right)$. This 


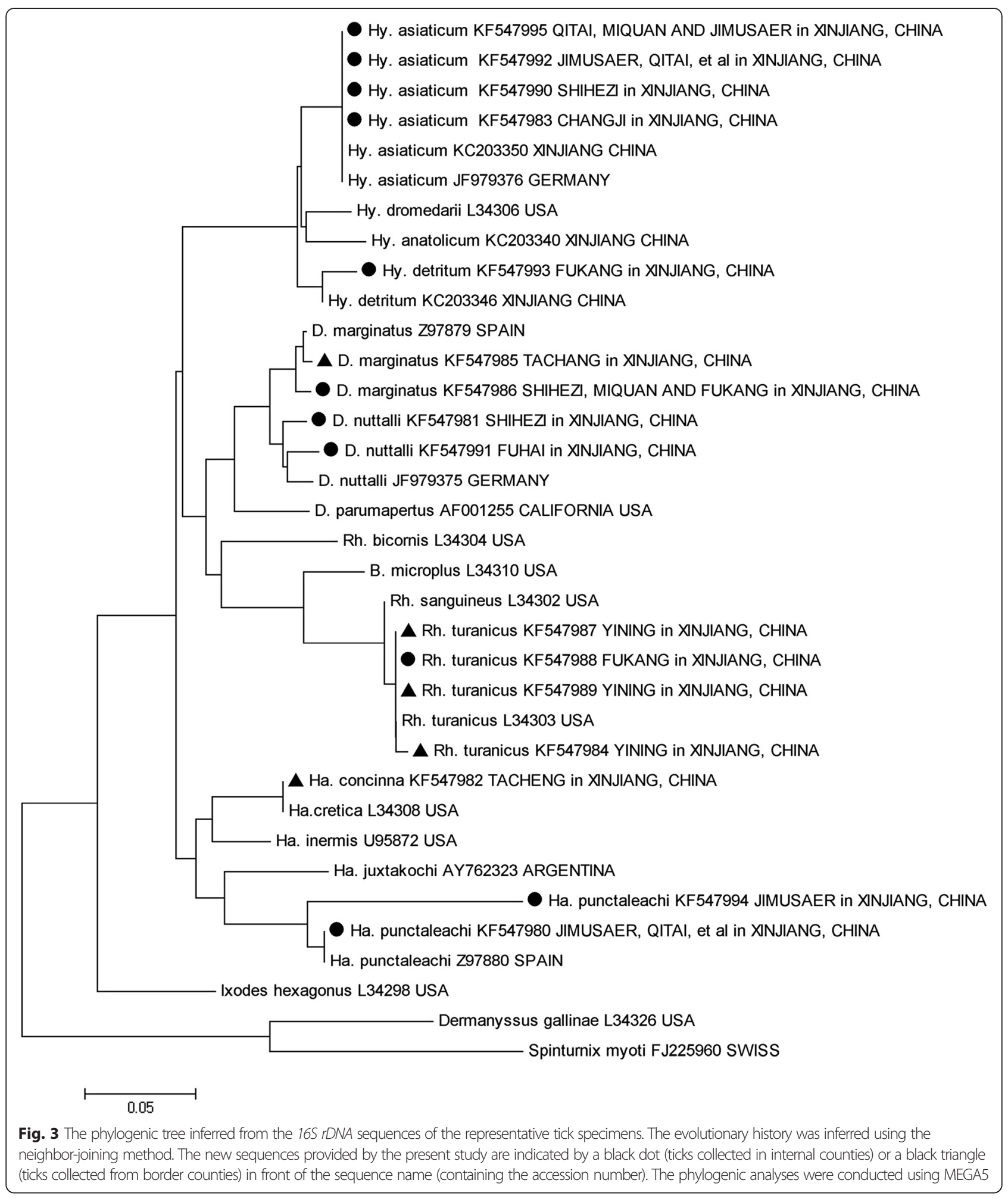

shift in the latitudinal distribution shows that the high temperatures in winter and the increased precipitation in spring and autumn may favour the survival, activity and development of Rh. turanicus. This phenomenon appears to be coincident with the spatial changes observed in I. ricinus in Europe due to the effects of climate change $[18,19]$.

Third, the geographical distribution of $H$. punctata and D. marginatus has expanded. In China, $H$. punctata is only distributed in Xinjiang and Gansu Province and 


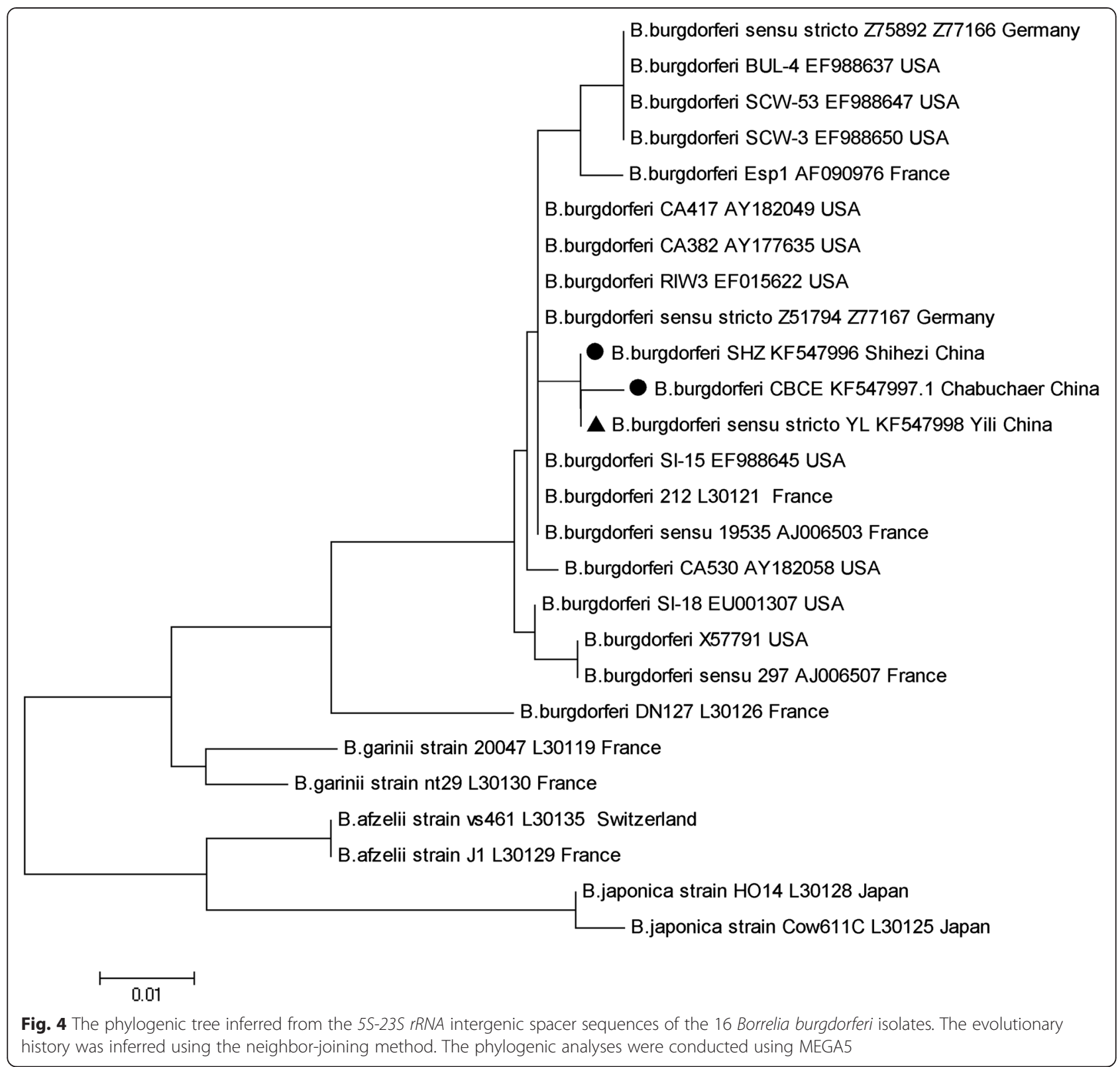

ranges from 580 to 1700 m.a.s.l. Its habitat varies, including meadows, conifer-broadleaf forests, thickets, and semi-desert. In this study, H. punctata was found to be the second most abundant and the most widespread tick species compared with a report for the period from 1973 to 1985 . We collected $H$. punctata from Jimusaer, Qitai, Mulei, Fukang, Karamay, Shihezi, Shawan, Fuhai, Qinghe, and Tacheng Counties. Additionally, in China, D. marginatus is distributed throughout Xinjiang and ranges from 300 to 1600 m.a.s.l. Its habitats include semi-desert, saline-alkali soil, grasslands and thickets. D. marginatus used to be scattered throughout Yining, Tacheng, Karamay and Aertai Countries; however, in our study, we collected the species in Fukang, Miquan, Shihezi and Shawan Counties. These results strongly suggest that $H$. punctata and $D$. marginatus have expanded their range in the last three decades, particularly into the northern parts of Xinjiang. More studies are needed to further understand the phenomena above. For example, the survival rates of the eggs, larvae, nymphs and adult ticks of $I$. persulcatus, $H$. punctata and D. marginatus should be assessed under different RHs and environmental temperatures.

The analyses of the mitochondrial $16 S$ rDNA sequences were not conclusive but did provide support regarding the genetic similarities or diversity of several of the special tick species in some of the regions, which may have resulted 
from geographic separation and historical international livestock trade. Hy. asiaticum and Ha. punctata from Jimasa, Qitai, Mulei, Fuhai, Shihezi, Shawan, Karamay, Qinghe, Tacheng and Chabuchaer were found to share common 16S rDNA sequences (KF547992 and KF547980). These results show that these tick species are indigenous and evolved from the same lineage. In contrast, with the long-distance movement of livestock during periods of historical commercial trade, "foreign" ticks (tick species with different lineages) were introduced into some of the regions. Therefore, identical tick species from the same region showed genetic diversity, such as $\mathrm{Hy}$. asiaticum from Shihezi County (KF547992 and KF547990) and Rh. turanicus from Yining County (KF547984, KF547987 and KF547989).

Additionally, a high prevalence of tick-borne diseases (TBDs), such as Crimean-Congo hemorrhagic fever, borreliosis, and tick-borne encephalitis, exists in Xinjiang, and some tick-borne pathogens have appeared in the adjacent countries [20-23].

Borreliosis, which is caused by Borrelia burgdorferi sensu lato, is an important endemic zoonosis with a distribution closely related to the major ixodid tick vectors $[24,25]$. Of the 109 species of ticks identified in China, B. burgdorferi has been isolated from nine ixodid ticks, including I. persulcatus, I. granulatus, I. acutitarsus, $H$. longicornis, $H$. bispinosa, $H$. concinna, $H$. formosensis, Boophilus microplus and D. silvarum [26, 27]. We attempted to isolate Borrelia pathogens from the predominant tick species for the following reasons: i) borreliosisis prevalent in domestic animals in Xinjiang [4, 28]; ii) Liu et al. first detected $5 \mathrm{~s}-23 \mathrm{~s}$ rRNA intergenic spacer and flagllin gene of B. burgdorferi sensu stricto from livestock blood samples in the Ili and Altay Districts in northern Xinjiang in 2013; and iii) I. persulcatus, which is the vector for $B$. burgdorferi, has not been identified from the domestic animal species we sampled. Ultimately, we isolated B. burgdorferi sensu stricto from Hy. Asiaticum asiaticum, H. punctata, D. marginatus and $R h$. Turanicus we collected from domestic animals. This result shows that $H y$. asiaticum, $H$. punctata, D. marginatus and Rh. turanicus are carriers of B. burgdorferi sensu stricto and act as potential vectors for livestock borreliosis. Although our present research showed that 5S-23S rRNA intergenic spacer sequence of $B$. burgdorferi was positively detected from Hy. Asiaticum, H. punctata, D. marginatus and Rh. turanicus that were infesting the domestic animals we sampled, future work, such as virulence tests of tick bites in animals and transmission experiments, should be performed to further confirm whether these tick species are vectors for $B$. burgdorferi sensu stricto. Moreover, the seroprevalence and level of bacterial isolation from domestic animals should be continuously monitored.

\section{Conclusions}

In this study, we collected 5257 adult ticks from the sheep, cattle, horses and camels from 19 sampling sites in 14 counties in Xinjiang along the New Eurasian Continental Bridge, which is the area adjacent to Mongolia and Kazakhstan. The adult ticks belonged to four genera and seven species. We found that Hy. Asiaticum asiaticum, $H$. punctata, D. nuttalli, D. marginatus, and $R$. turanicus are the dominant tick species during the peak activity season of adult ticks in northern Xinjiang; this differs from the dominant tick species reported three decades ago. This change is likely due to the local climate change, which has become warmer and wetter. The phylogenetic tree indicates that certain tick species exhibit genetic similarities or diversity, which may be related to geographic separation and historical international livestock trade. Additionally, B. burgdorferi sensu stricto was isolated from Hy. Asiaticum asiaticum, $H$. punctata, D. marginatus and Rh. turanicus from domestic animals for the first time in northern Xinjiang. These four tick species may act as potential vectors for livestock borrelia infection in Xinjiang.

\section{Additional files}

Additional file 1: Table S1. Geographic information for the 19 sampling sites at 14 surveyed counties. (DOC $55 \mathrm{~kb}$ )

Additional file 2: PCR protocol for the detection of 110 representative tick specimens and Borrelia isolates, northern Xinjiang, China. (DOC 50 kb)

\section{Competing interests}

The authors declare that they have no competing interests.

\section{Authors' contributions}

C-FC conceived the study and participated in its design. Y-ZW, L-MM, LZ, $Z-Q L, Y W, J-Y D, R-Y C, J X, W-J Z, L Y$ and $W$-ZZ performed the tick collection. $\mathrm{L}-\mathrm{XL}$ conducted the morphological analysis. $\mathrm{KZ}$ and R-FS conducted the molecular analyses. KZ submitted all of the sequences to GenBank and performed the phylogenetic tree analysis. W-HL and M-HY analysed the geographic information using Maplnfo. Y-ZW wrote the first draft of the manuscript. Y-ZW, KZ and R-FS revised the manuscript. All the authors approved the final version of the manuscript.

\section{Acknowledgments}

This research was supported by grants from the National Science

\&Technology Pillar Program (2013BAI05B05), Co-innovation Center for the High Incidence of Zoonotic Disease Prevention and Control in Western China (2013-179), and the National Nature Science Foundation of China (31060334 and 31260596).

\section{Author details}

${ }^{1}$ School of Medicine, Shihezi University, 832003 Shihezi, Xinjiang, Peoples Republic of China. ${ }^{2}$ Institute of Agricultural and Rural Development, Pingdingshan University, 467000 Pingdingshan, Henan, Peoples Republic of China. ${ }^{3}$ School of Agriculture, Shihezi University, 832003 Shihezi, Xinjiang, Peoples Republic of China. ${ }^{4}$ Jinan Center for Disease Control and Prevention, 250021 Jinan, Shandong, Peoples Republic of China. ${ }^{5}$ School of Animal \& Science, Shihezi University, 832003 Shihezi, Xinjiang, Peoples Republic of China. ${ }^{6}$ Veterinary Research Institute, Xinjiang Academy of Animal Sciences, 830000 Urumqi, Xinjiang, Peoples Republic of China. ${ }^{7}$ lli Center of Animal Disease Control and Diagnosis, 835000 Ili, Xinjiang, Peoples Republic of 
China. ${ }^{8}$ Xijiang Entry - Exit Inspection and Quarantine Authority of the PRC, 830063 Urumqi, Xinjiang, Peoples Republic of China. ${ }^{9}$ First Affiliated Hospital of School of Medicine, Shihezi University, 832008 Shihezi, Xinjiang, Peoples Republic of China. ${ }^{10}$ Genecology Research Center, University of the Sunshine Coast, Queensland 4558, Australia.

Received: 24 April 2015 Accepted: 29 July 2015

Published online: 04 September 2015

\section{References}

1. Masuzawa T. Terrestrial distribution of the Lyme borreliosis agent Borrelia burgdorferi sensu lato in East Asia. Jpn J Infect Dis. 2004;57(6):229-35.

2. Hao Q, Hou X, Geng Z, Wan K. Distribution of Borrelia burgdorferi sensu lato in China. J Clin Microbiol. 2011;49(2):647-50.

3. Takada N, Masuzawa T, Ishiguro F, Fujita H, Kudeken M, Mitani H, et al. Lyme disease Borrelia spp. in ticks and rodents from northwestern China. Appl Environ Microbiol. 2001;67(11):5161-5.

4. Liu G, Tian Z, Xie J, Luo J, Tian M. Borrelia burgdorferi bacteria in domestic animals china. Epidemiol. 2013;3(133):2161-1165.

5. Meng M, Pu C-I. Analysis on regional difference of using efficiency of building land-a case study of Xinjiang. Technoeconom Manag Res. 2011;11:007.

6. Zhang ZW, Yang FX, Wu JL, Zhou J, Yin HY. Spatial distribution patterns and type structure of the deserts in Xinjiang. Arid Zone Research. 2014;31(4):763-70.

7. Chen Z, Yang X, Bu F, Yang X, Yang X, Liu J. Ticks (acari: ixodoidea: argasidae, ixodidae) of China. Exp Appl Acarol. 2010;51(4):393-404.

8. Kong Zhaomin CG, Genyuan J, Peiyu S, Xinrong Z, Xuegin L. Investigations on ticks and tick-borne natural focal infections in Xinjiang. Bull Endem Dis. 1987;2(3):1-3.

9. Walker AR, Bouattour A, Camicas J, Estrada-Pena A, Horak I, Latif A, et al. Ticks of domestic animals in Africa: a guide to identification of species. UK: Bioscience Reports; 2003.

10. Estrada-Peña A, Bouattour A, Camicas J-L, Walker AR. Ticks of domestic animals in the Mediterranean region: a guide to identification of species. SPN: University of Zaragoza; 2004

11. Black WC, Piesman J. Phylogeny of hard- and soft-tick taxa (Acari: Ixodida) based on mitochondrial 165 rDNA sequences. Proc Natl Acad Sci U S A. 1994;91(21):10034-8.

12. Burgdorfer W. Discovery of the Lyme disease spirochete and its relation to tick vectors. Yale J Biol Med. 1984;57(4):515-20.

13. Larkin M, Blackshields G, Brown N, Chenna R, McGettigan PA, McWilliam H, et al. Clustal W and Clustal X version 2.0. Bioinformatics. 2007;23(21):2947-8.

14. Tamura K, Peterson D, Peterson N, Stecher G, Nei M, Kumar S. MEGA5: molecular evolutionary genetics analysis using maximum likelihood, evolutionary distance, and maximum parsimony methods. Mol Biol Evol NLM. 2011;28(10):2731-9.

15. Saitou N, Nei M. The neighbor-joining method: a new method for reconstructing phylogenetic trees. Mol Biol Evol NLM. 1987:4(4):406-25.

16. Yu X, Ruiyu Y, Zijian Z, Xinru C. Faunal investigation on the ticks in Boertala and Yili distriction of Xinjiang. China Endemic Dis Bull. 1993;8(2):39-43.

17. Xin Yu RY, Zhang Z, Chen X. Faunal investigation on the ticks in Boertala and Yili distriction of Xinjiang, China. Endemic Dis Bull. 1993;8(2):5

18. Jore $\mathrm{S}$, Vanwambeke SO, Viljugrein $\mathrm{H}$, Isaksen $\mathrm{K}$, Kristoffersen $\mathrm{AB}$, Woldehiwet $Z$, et al. Climate and environmental change drives Ixodes ricinus geographical expansion at the northern range margin. Parasit vectors. 2014;7:11.

19. Jaenson TG, Jaenson DG, Eisen L, Petersson E, Lindgren E. Changes in the geographical distribution and abundance of the tick Ixodes ricinus during the past 30 years in Sweden. Parasit vectors. 2012;5:8.

20. Dedkov VG, Markelov ML, Gridneva KA, Bekova MV, Gmyl AP, Kozlovskaya LI, et al. Prevalence of Kemerovo virus in ixodid ticks from the Russian Federation. Ticks Tick Borne Dis. 2014;5(6):651-5

21. Karimov SK, L'Vov DK, Rogovaia SG, Kiriushchenko TV, Ukbaeva TD. Isolation of the Karshi virus from Hyalomma asiaticum ticks in Alma-Ata Province, Kazakh SSR. Med Parazitol (Mosk). 1978;47(3):50-1.

22. Al'khovskii SV, L'Vov DK, Shchelkanov M, Shchetinin AM, Deriabin PG, L'Vov DN, et al. Genetic characterization of the Batken virus (BKNV) (Orthomyxoviridae, Thogotovirus) isolated from the Ixodidae ticks Hyalomma marginatum Koch, 1844 and the mosquitoes Aedes caspius Pallas, 1771, as well as the Culex hortensis Ficalbi, 1889 in the Central Asia. Vopr Virusol. 2014;59(2):33-7.
23. L'Vov DK, Al'khovskii SV, Shchelkanov M, Shchetinin AM, Deriabin PG, Aristova VA, et al. Taxonomic status of the Tyulek virus (TLKV) (Orthomyxoviridae, Quaranjavirus, Quaranfil group) isolated from the ticks Argas vulgaris Filippova, 1961 (Argasidae) from the birds burrow nest biotopes in the Kyrgyzstan. Vopr Virusol. 2014;59(2):28-32.

24. Fryxell RT, Steelman CD, Szalanski AL, Kvamme KL, Billingsley PM, Williamson PC. Survey of Borreliae in ticks, canines, and white-tailed deer from Arkansas, U.S.A. Parasit vectors. 2012;5:139.

25. Schwarz A, Honig V, Vavruskova Z, Grubhoffer L, Balczun C, Albring A, et al. Abundance of Ixodes ricinus and prevalence of Borrelia burgdorferi s.l. in the nature reserve Siebengebirge, Germany, in comparison to three former studies from 1978 onwards. Parasit vectors. 2012;5:268.

26. Niu Q, Guan G, Yang J, Fu Y, Xu Z, Li Y, et al. Detection and differentiation of Borrelia burgdorferi sensu lato in ticks collected from sheep and cattle in China. BMC Vet Res. 2011:7:17.

27. Niu QLYH, Luo JX. Progress on Lyme disease in China. Prev Vet Med. 2009:30:5.

28. Qi Z, Dong W, Zhijun Y, Yuanzhi W, Kanglin W, Yonggang Q. Seroepidemiological Investigation to Cattle and Sheep Lyme Disease in State Farm 150 of Xinjiang BINGTUAN. Journal of Shihezi University (Natural Science). 2006;24(4):454-59.

\section{Submit your next manuscript to BioMed Central and take full advantage of:}

- Convenient online submission

- Thorough peer review

- No space constraints or color figure charges

- Immediate publication on acceptance

- Inclusion in PubMed, CAS, Scopus and Google Scholar

- Research which is freely available for redistribution 